\title{
CHROMOSPHERIC AND CORONAL HEATING MECHANISMS
}

\author{
P.Ulmschneider ${ }^{1}$ and U.Narain ${ }^{2}$ \\ 1 Insti. f. Theor. Astrophys., Im Neuenheimer Feld \\ 561, D-6900 Heidelberg, W.Germany \\ 2 Astrophysics Research Group, Meerut College, \\ Meerut-250003, India
}

Stellar chromospheres lose energy, predominantly, via radiation and coronae via conduction, radiation and stellar wind flows. To prevent these hot layers from rapidly cooling down to photopsheric temperature some source of heating must be present.

Various heating mechanisms have been proposed to date. For details see Narain and Ulmschneider (1989) and references contained therein.

This study leads us to conclude that in late-type stars short-period waves seem to contribute a basic but weak background heating, independent of rotation. In rotating late-type stars magnetic field related heating is dominant. Slow mode and longitudinal tube waves seem to heat low and middle chromosphere via shocks readily. Locally produced fast mode waves may heat coronal loops via shocks and Landau damping. Transverse and torsional Alfven waves can also heat coronal loops via resonant absorption, phase mixing, nonlinear mocie ccupling and Landau damping. Alfvenic surface waves can heat these loops via resonant absorption in thin sheaths. The loops can also be heated via current (magnetic field) dissipation through ancmalous joule heating and reconnection.

Coronal holes are heated, primarily, by dissipation of shear Alfven waves via phase mixing, nonlinear mode coupling turbulent and Landau damping.

One of the authors (U.N.) is very much thankful to Prof.Vinod Krishan and other organisers for their financial help and encouragement.

Narain,U. and Ulmschneider,P. : 1989 Space Sci. Rev. (In Press).

97

E. R. Priest and V. Krishan (eds.), Basic Plasma Processes on the Sun, 97.

- 1990 IAU. Printed in the Netherlands. 